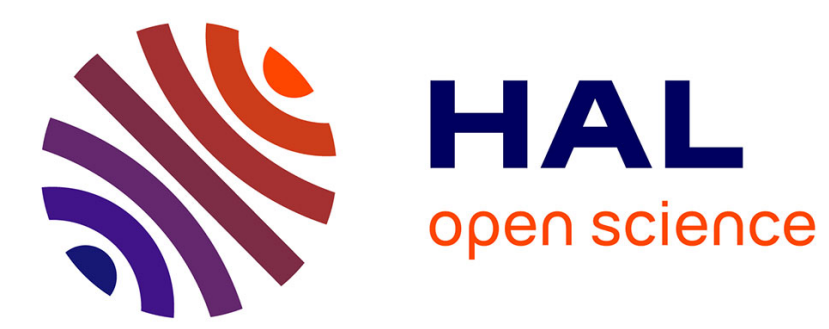

\title{
Retour à Lergnes
}

Elie Guéraut

\section{- To cite this version:}

Elie Guéraut. Retour à Lergnes. Genèses. Sciences sociales et histoire, 2021, n 122 , pp.107 - 126. 10.3917/gen.122.0107. hal-03404697

\section{HAL Id: hal-03404697 https://hal.uca.fr/hal-03404697}

Submitted on 31 Oct 2021

HAL is a multi-disciplinary open access archive for the deposit and dissemination of scientific research documents, whether they are published or not. The documents may come from teaching and research institutions in France or abroad, or from public or private research centers.
L'archive ouverte pluridisciplinaire HAL, est destinée au dépôt et à la diffusion de documents scientifiques de niveau recherche, publiés ou non, émanant des établissements d'enseignement et de recherche français ou étrangers, des laboratoires publics ou privés. 
Titre : Retour à Lergnes

Sous-titre : Les mobilités professionnelles contrariées de jeunes diplômées des Beaux-arts

Auteur : Elie Guéraut, Lescores, Université Clermont-Auvergne, chercheur invité au sein de l'unité List de l'Ined.

Adresse postale : 11 rue de l'Oratoire, 58000 Nevers

Numéro de téléphone : 0641758510

Adresse email : Elie.gueraut@gmail.com

Résumé : A partir du cas de jeunes diplômées d'écoles d'art dont les aspirations à devenir artistes-plasticiennes se heurtent aux droits d'entrée du marché du travail artistique, cet article propose une analyse de la production d'un désajustement durable entre mobilités professionnelles et aspirations subjectives. La première partie de l'article s'attache à rendre compte du processus de production de ces désillusions, à la croisée de cette mobilité professionnelle empêchée et d'un retour contraint à l'espace d'origine, une ville moyenne en déclin. La deuxième partie revient sur le réajustement et l'abandon des vocations artistiques au sein de deux « univers de consolation », la scène associative et le monde privé conjugal.

Présentation de l'auteur : Elie Guéraut est sociologue au Lescores et enseignant à l'université Clermont-Auvergne. Il est également chercheur invité au sein de l'unité List à l'Ined. Il travaille actuellement sur les migrations résidentielles et les transformations sociales et politiques des villes petites et moyennes en marge des grandes agglomérations. Il a récemment publié «Le mépris comme réassurance sociale. Une petite bourgeoisie culturelle confrontée à son déclin » (Sociétés Contemporaines, vol. 4, n 120, 2020). 


\section{Retour à Lergnes. Les mobilités professionnelles contrariées de jeunes diplômées des Beaux-arts}

\section{Introduction}

Les années 1990 ont été le théâtre d'une ouverture inédite de l'enseignement supérieur aux classes populaires, qui ne s'est toutefois pas accompagnée d'une disparition des inégalités de destins sociaux et professionnels des étudiants selon leur origine sociale (Chauvel 1998 ; Merle 2009). Si un tel constat est d'abord formulé sur la base de données quantitatives, différentes enquêtes ethnographiques se sont attachées à rendre compte des « désillusions » des primo-accédants à l'université, qu'elles soient consécutives aux échecs scolaires rencontrés (Beaud 1997, 2002) ou qu'elles résultent des difficultés à tirer profit des titres scolaires obtenus (Hugrée 2010 ; Pasquali 2010 ; Truong 2015). Ces déboires concernent tout particulièrement les jeunes de classes populaires qui aspirent à intégrer le marché du travail artistique, dont les « droits d'entrée » reposent sur des ressources culturelles et relationnelles qui leur font défaut (Mauger 2007), tandis que la prégnance de l'idéologie du «talent » invisibilise le poids de l'héritage (Dubois 2011 : 94). Les difficultés qu'ils peuvent rencontrer à faire de la production artistique ou de l'intermédiation culturelle leur métier sont alors interprétées comme preuve d'une incompétence propre, qui s'impose d'autant plus violemment que les filières qui les y dirigent sont caractérisées par un fort pouvoir d'acculturation. L'abandon des vocations artistiques, qui se traduit par une mobilité professionnelle horizontale (du marché du travail artistique vers d'autres secteurs d'activité), nourrit alors un sentiment de déclassement, à plus forte raison lorsqu'il s'accompagne, comme dans les cas qui sont traités dans cet article, d'un retour à un espace d'origine stigmatisé, Lergnes, une ville moyenne du centre de la France sujette au déclin urbain ${ }^{1}$.

\footnotetext{
${ }^{1}$ Afin de préserver l'anonymat des enquêtés, le nom de la ville a été changé. Précisons seulement que cette ville présente des caractéristiques communes aux villes moyennes en déclin du centre de la France : présence importante et déclinante de l'emploi public, décroissance démographique, paupérisation des habitants, dégradation de la valeur économique et symbolique de l'espace, distance importante aux grandes agglomérations.
} 
A travers l'analyse des premières mobilités professionnelles et des difficultés d'accès à ce marché du travail spécifique de jeunes diplômées des écoles des Beaux-arts de Lyon et de Marseille, cet article entend discuter de la question des désajustements entre les aspirations socio-résidentielles que font naître une mobilité scolaire objective et une ascension professionnelle qui ne se produit pas. A quelles conditions et sur quelles scènes sociales se construit un tel décalage? Que doit cette situation aux rapports de classe, de genre, et aux effets objectifs et subjectifs des «appartenances territoriales » (Laferté et Weber 2019) ? Comment s'articulent dans ce retour à l'espace d'origine mobilité sociale (objective et subjective), mobilité professionnelle et mobilité géographique ? La démarche ethnographique constitue la méthode la plus à même d'aborder ces questions pour au moins deux raisons. En premier lieu, du fait qu'elle permette d'observer sur le temps long les mobilités « en train de se faire » (Pagis et Pasquali 2016). Elle offre ainsi les moyens d'une analyse fine du processus à travers lequel les aspirations initiales vont, au fil des années, être réajustées, jusqu'à parfois être abandonnées, venant contrarier le sentiment de réussite sociale. Ensuite, car cette méthode d'enquête donne la possibilité de suivre les enquêtés d'une scène sociale à l'autre et ainsi d'apprécier comment la mobilité subjective est façonnée par des influences parfois contradictoires, qui s'exercent sur les scènes résidentielle, professionnelle, associative et militante, ainsi que conjugale et familiale.

L'enquête ethnographique sur laquelle s'appuient les résultats présentés dans cet article a été conduite de 2010 à 2018 à Lergnes. Celle-ci portait à l'origine sur les migrations résidentielles des étudiants originaires des espaces en marge des grandes agglomérations, et interrogeait plus particulièrement le cas de ceux qui rentrent à proximité de leur entourage à l'issue de cette expérience. Dans le cadre de cette enquête, les trajectoires de 42 jeunes diplômés de l'enseignement supérieur ayant fait l'expérience d'un retour dans leur espace d'origine ont été reconstituées et analysées, par le biais d'entretiens, d'échanges informels et d'observations, 
permettant un suivi longitudinal sur plusieurs années. Cette enquête ethnographique a ensuite été «armée» par des traitements statistiques portant sur les mobilités résidentielles des étudiants et jeunes diplômés du supérieur (à partir des recensements de la population de l'Insee) ainsi que sur les vœux d'orientation des bacheliers du département rural dont Lergnes est la préfecture (à partir de la base Admission Post-Bac du rectorat de l'Académie). Il m'a été ainsi possible de situer ce cas ethnographique à la fois dans l'espace local et national, et ainsi de faciliter la montée en généralité des résultats (Weber 1995).

Les cas qui seront analysés dans cet article ont tous été rencontrés dans le cadre d'une observation participante au sein de deux associations culturelles : la première assurant la gestion d'un café organisant concerts, débats et expositions d'art et comprenant 12 membres; la deuxième celle d'une galerie d'art contemporain dirigée par un couple. Les jeunes diplômés qui s'engagent dans ces deux associations présentent plusieurs caractéristiques communes, qui les distinguent de la population étudiante saisie à l'échelle locale et nationale. D'une part, ils sont très majoritairement d'origine populaire ${ }^{2}(12 / 14)$. Ils ont, avant de rentrer à Lergnes, poursuivis des études supérieures au sein de filières sélectives de production ou d'intermédiation culturelles (8/14), caractérisées par une position dominée au sein des régions supérieures de l'espace de l'enseignement supérieur (Convert 2010 : 22), où s'observe une surreprésentation des enfants des classes supérieures ${ }^{3}$. Il s'agit en outre en majorité de femmes (10/14), en raison de leur forte présence dans ces formations (Erb 2018), mais aussi du fait que ce phénomène de « retour qualifié » les concerne plus volontiers que les hommes (Guéraut et Jedlicki 2021). Bien

\footnotetext{
${ }^{2}$ Suivant Siblot et al., cette définition des classes populaires intègre les petits artisans et commerçants, les employés et les ouvriers (2015).

${ }^{3}$ A l'échelle du département d'enquête, l'orientation vers une école d'art représente un vœu rare $(4,2 \%$ de l'ensemble des vœux) et peu plébiscité par les classes populaires : les enfants d'employés et d'ouvriers représentent respectivement $20,6 \%$ et $6,5 \%$ des effectifs (contre $23,5 \%$ et $18,8 \%$ de l'ensemble des bacheliers du département ayant formulé un vœu d'orientation dans le supérieur) tandis que la part des enfants de cadres et de professions intellectuelles supérieures y est maximale à 31,5\% (contre 16,6\% dans l'ensemble).
} 
davantage que pour les autres jeunes diplômés interrogés, ce retour s'organise pour elles sous de fortes contraintes et constitue à ce titre une expérience déceptive, venant alimenter un sentiment de $«$ lergnose $»^{4}$, comme nous le verrons dans la première partie de cet article. Il vient par ailleurs accélérer un retrait du marché du travail artistique qu’elles avaient pu intégrer «par le bas $»^{5}$ au sein des grandes agglomérations, celui-ci se résumant à Lergnes à quelques dizaines de postes déjà pourvus et, de ce fait, jugés inaccessibles. En conséquence, le retour va de pair avec des mobilités professionnelles à destination de secteurs de l'emploi public peu concurrentiels, l'éducation et le travail social pour l'essentiel, dans la très grande majorité des cas sous le statut de contractuel et à temps partiel (contraint).

L'analyse proposée dans cet article s'appuiera principalement sur la comparaison des trajectoires de deux jeunes femmes, Alice et Sandy ${ }^{6}$, saisies dans leur dimension professionnelle, géographique et matrimoniale. Ce choix tient pour une part aux relations d'enquête privilégiées qui ont été tissées avec elles, permettant un suivi longitudinal approfondi sur plusieurs années. En outre, leur cas est remarquable des aspirations et des déceptions d'une fraction particulière de la jeunesse, féminine, caractérisée par des origines populaires et rurales et par un désir d'émancipation sociale tourné vers les mondes de l'art. Il donne ainsi les moyens de mettre au jour les conditions de production d'un désajustement durable entre les mobilités professionnelles objectives et les aspirations subjectives qui, s'il apparaît ici exacerbé pour des

\footnotetext{
${ }^{4}$ Le terme original, qui vise à qualifier l'absence de perspectives professionnelles et matrimoniales qu'offrirait leur position socio-résidentielle actuelle, est forgé par l'association du nom du département et du suffixe «ose » («[nom du département]-ose »). Le néologisme indigène ainsi créé se rapproche volontairement d'un terme médical désignant une pathologie psychologique. Il ne peut être révélé sans compromettre l'anonymat des enquêtés. Précisons que celui-ci n'est pas employé par ces seules étudiantes de retour, il l'est plus généralement par l'ensemble des classes supérieures qui partagent la reconnaissance de l'assignation de leur espace résidentiel au bas de la hiérarchie symbolique des territoires.

${ }^{5}$ Pour l'essentiel par le biais de contrats courts et mal rémunérés dans l'intermédiation culturelle.

${ }^{6}$ Afin de garantir l'anonymat des enquêtés les prénoms des enquêtés ont été changés. Dans deux des quatre cas discutés dans cet article, les prénoms anglo-saxons originaux ont été remplacés par des prénoms cultivant une même origine linguistique (Sandy et Antony). Ce choix traduit un souci de rappeler leurs origines populaires, qui, comme on le verra, ne sont pas étrangères aux déboires rencontrés dans leur tentative d'intégrer le marché du travail artistique.
} 
raisons que l'on s'attachera à éclaircir, concerne à des degrés moindres la majorité des diplômés rentrés à Lergnes. Au total, cet article propose ainsi une ethnographie de la construction de la mobilité subjective sur le temps long, dans sa dimension spatiale et appréciée depuis différentes scènes sociales. Il s'agit en outre de varier les niveaux d'analyse, en interrogeant à la fois ce que ces mobilités professionnelles doivent aux conditions sociohistoriques qui participent à les rendre possible (ou impossible), ainsi que les conséquences des désajustements entre mobilité objective et aspirations subjectives, qui s'expriment à l'échelle individuelle sous la forme d'un sentiment de déclassement pérenne.

\section{La fermeture progressive du marché du travail artistique}

\section{Socialisation universitaire et vocations artistiques}

En dépit de leurs origines populaires et autochtones, les jeunes femmes dont il est question dans cet article ont connu l'expérience d'une émigration étudiante de plusieurs années, qui leur a permis d'obtenir un diplôme de niveau $\mathrm{Bac}+5$. Le caractère exceptionnel de cette réussite scolaire se doit d'être souligné. On ne peut en effet tout à fait saisir la mesure des espoirs qu'elles nourrissent pendant et à l'issue de leurs études supérieures (et donc les ressentiments engendrés par le retour) sans rapporter leurs aspirations professionnelles et sociales au champ des possibles qui s'offrent à elles, au sein duquel la poursuite d'études supérieures, même dans une filière non-sélective et à destination des grandes agglomérations les plus proches, constitue une expérience étrangère et improbable, que ni les parents ni les autres membres de la fratrie n'ont connue.

Alice, une jeune femme née en 1983 et rentrée à Lergnes en 2010, est ainsi la première de sa lignée familiale à poursuivre des études supérieures. Née d'un père espagnol arrivé à sept ans dans le département, aujourd'hui commercial itinérant de systèmes de tuyauterie, et d'une mère autochtone qui a été toute sa vie aide à domicile, Alice grandit dans une commune rurale à proximité de Lergnes avec son frère cadet (employé dans la grande distribution lorsque je 
rencontre la jeune femme en 2013). Elle rejoint le lycée fréquenté par la petite bourgeoisie culturelle locale ${ }^{7}$, faisant le vœu d'options rares (LV3 russe et option facultative musique), comme sa meilleure amie d'alors, une enfant d'enseignants rencontrée au collège. Alice se retrouve ainsi dans un contexte propice à l'émergence d'aspirations culturelles, qui prennent même en classe de terminale la forme d'une «vocation », celle de faire de la production d'œuvres d'art contemporain son métier. Son bac L obtenu (mention AB), elle prépare avec d'autres de ses camarades les concours d'entrée aux écoles des Beaux-arts et rejoint finalement celle de Lyon en 2002.

Alice va, durant les cinq années que compte sa formation aux Beaux-arts de Lyon, s'investir dans de nombreuses pratiques culturelles et sociabilités en lien avec sa formation. Elle se rend à des vernissages, des expositions, ou plus simplement partage un verre avec ses camarades de promotion. Elle se met en couple dès la première année avec un camarade de promotion, fils de professeurs du secondaire, qui l'encourage à poursuivre cette vocation à devenir artiste-plasticienne tout au long des cinq années que compte la formation. Alice parvient en quatrième année à exposer son travail avec l'aide d'une de ses professeurs dans plusieurs galeries lyonnaises. A l'issue de ses études, la jeune femme cumule ainsi les caractéristiques qui rendent crédible son entrée au sein du marché du travail artistique : un diplôme distinctif d'une école d'art sélective (Naudier 2014 : 203), des expériences professionnelles au sein de son univers d'élection (ibid., p. 191), l'engagement dans une relation stable depuis plusieurs années avec un camarade de promotion mieux doté, qui partage les mêmes aspirations culturelles et l'encourage dans sa vocation (Buscatto 2008 : 97), ainsi que la localisation de sa

\footnotetext{
${ }^{7}$ Cet établissement est celui où les enfants de cadres du secteur public sont les plus fréquents. Ils représentent $11,2 \%$ des élèves contre 4,6 \% en moyenne sur l'ensemble des lycées de l'agglomération lergnoise (source : BCS 2013, Depp).
} 
sociabilité dans l'espace d'une grande agglomération, qui participe à renforcer ses relations sociales sur le marché du travail artistique lyonnais (Buscatto 2015).

Cependant, lorsqu'elle obtient son diplôme et perd le statut d'étudiante, elle peine à revendiquer celui d'artiste-plasticienne. Malgré une exposition personnelle à la sortie de l'école dans une galerie lyonnaise et sa présence au salon d'art contemporain d'une capitale européenne l'année suivante, elle ne tire aucun bénéfice de ces expériences. Son incapacité en ces occasions où elle n'évolue plus dans l'entre soi de l'école, à faire preuve de « mondanité » (pour reprendre ses termes), c'est-à-dire à convertir son capital culturel incorporé en capital social lors des interactions codifiées qui peuvent se nouer dans ces lieux de sociabilité, lui apparaît alors comme un facteur qui compromet sa vocation. C'est à cette période qu'elle quitte son compagnon rencontré en première année, après plus de six ans de vie commune, décision qu'elle justifie rétrospectivement par le « ras-le-bol» d'être systématiquement vue comme «la copine de », assignation genrée qui fragilise sa légitimité propre à assumer ce statut d'artiste. Il faut aussi ajouter, afin de comprendre pleinement les difficultés que rencontre Alice à rester à Lyon, la question des contraintes matérielles qui s'exercent sur elle. Même si la perte des bourses étudiantes est compensée par son inscription au RSA (elle vient alors d'avoir vingtcinq ans) ainsi que par des petits contrats de surveillance de galerie, cette précarité économique, pourtant largement partagée parmi les jeunes artistes (Mayaud et Jeanpierre 2020), devient de plus en plus contraignante à mesure que s'éloigne la perspective d'intégrer le champ de l'art contemporain qui la protégeait jusqu'ici du stigmate de la pauvreté ${ }^{8}$. S'ajoutent à cette instabilité économique plusieurs autres histoires d'amour éphémères et malheureuses qui la

\footnotetext{
${ }^{8}$ Cette précarité économique, loin d'être stigmatisante, constitue plutôt une marque d'inscription dans le style de vie de la «bohème» dans les régions de l'espace social où elle évolue et atteste ainsi d'une certaine forme «d'authenticité » (Mauger $2007: 260-261$ ), qu'elle continue de valoriser plusieurs années après cet épisode lorsque je l'interroge sur ce passé.
} 
conduisent finalement, un peu moins de trois ans après avoir été diplômée, à retourner à Lergnes.

L'échec qu'elle connaît dans sa tentative d'intégrer le champ de l'art contemporain tient ainsi à plusieurs carences et montre à quel point la détention d'un diplôme, fut-il obtenu dans une école d'art sélective, n'a rien d'un sésame qui lui ouvrirait les portes du milieu qu'elle aspire à rejoindre. Cet échec tient à l'absence de certaines « dispositions acquises dans la fréquentation des œuvres et la pratique professionnelle (et donc pas seulement dans le cadre scolaire)» (Dubois $2011: 44-45)$ ainsi que d'un déficit de «capital spécifique » (Mauger 2006), propre au champ de l'art contemporain. Il faut également mentionner la modestie de son capital social efficient dans ce champ : si une de ses professeurs lui offre à deux reprises l'occasion de « sortir son travail de l'école », aucune autre proposition ne lui sera faite dès lors qu'elle quitte les Beaux-arts. Enfin, soulignons combien son entrée sur le marché du travail artistique lyonnais est à la fois fragile et peu distinctive. Dans l'incapacité de s'inscrire dans des activités de production artistique rémunérées (résidences d'artiste, contrats avec des galeries, etc.), Alice est en effet contrainte à s'en tenir à quelques vacations ponctuelles de surveillance de galeries. Ainsi, l'échec de la jeune femme à intégrer le marché du travail artistique résulte de sa confrontation à ces « droits d'entrée tacites », dont plusieurs recherches montrent qu'ils portent davantage préjudice aux jeunes de classes populaires et aux femmes (Buscatto $2008: 88$; Ravet 2006 : 157-160), en particulier dans le champ de l'art contemporain (Pasquier 1983).

A son retour à Lergnes, Alice vit d'abord quatre mois chez un couple d'amis dans une petite commune de 5000 habitants à une trentaine de kilomètres de Lergnes, afin d'éviter une cohabitation avec sa mère qu'elle appréhende («ça aurait été trop la loose »). Durant cette période, elle sort très peu, regarde des séries, pleure quotidiennement, et dit être « incapable de réfléchir », « déprimée à mort». Ce retour s'accompagne ainsi d'un épisode qu'elle qualifie de 
«dépressif», trahissant une «angoisse du possible renvoi vers la position d'origine », également analysés par Pierre-Emmanuel Sorignet dans le cas de danseurs professionnels d'origine populaire contraints à la sortie du métier (2016). Ce sentiment est ici d'autant plus fort que la jeune femme s'inscrit dans une trajectoire scolaire caractérisée par une importante réussite, contrairement aux étudiants «demi-acculturés » par l'expérience d'une scolarité longue (Beaud 2002), dont la «socialisation scolaire et universitaire est inachevée» (Hugrée 2011). Il résulte d'un effet d'hystérésis, également analysé par Henri Eckert dans son travail sur le « désenchantement » des étudiants d'origine populaire (2011: 64), les aspirations et le style de vie adoptés durant ses années aux Beaux-arts à Lyon se trouvant soudainement désajustés à la réalité de sa position socio-résidentielle présente. La pérennisation de ce sentiment d'échec tient à deux facteurs que je me propose maintenant d'éclaircir : en premier lieu, l'exclusion du marché du travail artistique et l'accès à l'emploi public précarisé ; en second lieu, le fait que ce retour ait pour destination Lergnes, une ville moyenne en déclin du centre de la France.

\section{Retour à Lergnes et réajustement des vocations artistiques}

Le retour à Lergnes marque les débuts de la recherche d'un (nouvel) emploi. Ces jeunes diplômées font alors de cette ville leur «camp de base » à partir duquel elles prospectent dans la France entière à des emplois correspondant à la spécialisation et au niveau de leur diplôme, essentiellement des résidences d'artiste dans le cas de celles aspirant à s'inscrire dans la production artistique. Mais cette recherche est rarement concluante et ne donne qu'exceptionnellement lieu à des entretiens, qui se soldent par ailleurs toujours par un refus des employeurs. La prospection sur le marché local de l'emploi se fait en revanche plus fructueuse, au prix d'une sortie du marché du travail artistique.

Cet abandon progressif des vocations initiales s'explique par différents facteurs. Le retour a fragilisé la confiance de ces jeunes femmes qui ne se sentent plus «autorisées » 
(Ravet 2006 : 173) à gagner le marché du travail artistique. Ensuite, ce marché se résume localement à une vingtaine de postes déjà pourvus, pour l'essentiel relatifs à l'intermédiation culturelle et non à la production artistique ${ }^{9}$, de ce fait jugés inaccessibles et peu désirables. Par défaut, elles vont concentrer leurs recherches sur les institutions publiques et parapubliques susceptibles de reconnaître la valeur des titres scolaires rapportés de l'émigration étudiante, par ailleurs principaux employeurs de la zone d'emploi ${ }^{10}$. Les postes convoités sont ainsi ceux de la main gauche de l'Etat déconcentrée et décentralisée : professeurs, éducateurs, agents de catégorie A ou B de la FPT, etc. Si cette mobilité professionnelle horizontale, qui engage un retrait du marché du travail artistique pour le secteur éducatif ou le travail social, apparaît acceptable aux yeux de ces jeunes femmes, c'est qu'elle s'accompagne de la promesse d'une mobilité professionnelle ascendante (compte tenu de leurs origines sociales et des «petits boulots » exercés durant les études). Pourtant, la précarité et l'incertitude qu'elles y rencontrent contredisent rapidement cet espoir et, associées à l'abandon progressif des vocations artistiques, suscitent de profondes désillusions, comme je me le propose de le montrer en revenant au cas d'Alice à son retour à Lergnes.

À son arrivée, elle ne compte plus guère d'amis dans la région, à l'exception du couple chez qui elle s'installe durant ses quelques mois de «déprime». Elle loue ensuite un appartement dans le centre-ville et décide de reprendre contact avec Antony, un ancien camarade du lycée, enfant unique d'un ménage ouvrier dont la trajectoire sera détaillée dans la deuxième partie de cet article. Contentons-nous pour l'instant de préciser que le jeune homme connaît une mobilité professionnelle ascendante sur le marché du travail artistique, du ménage

\footnotetext{
${ }^{9}$ Pour l'essentiel, au sein des collectivité territoriales, d'associations culturelles subventionnées et des institutions culturelles d'Etat ou municipales.

${ }^{10}$ Les plus gros employeurs de la zone d'emploi sont, par ordre décroissant d'importance, le centre hospitalier (1 900 emplois), les collèges et les lycées (850 emplois), le conseil général (850 emplois) et la commune de Lergnes (800 emplois) (source : Insee, Siasp 2012).
} 
ouvrier lergnois duquel il est originaire jusqu'au poste de chargé de programmation dans un centre d'art contemporain de l'agglomération lergnoise qu'il occupe alors dans le cadre d'un CDI. Antony introduit Alice dans les sociabilités de la petite bourgeoisie culturelle locale, où elle retrouve l'occasion de présenter à nouveau son travail artistique. Elle y rencontre son compagnon actuel, Sébastien, technicien son (sous le statut d'intermittent du spectacle). Contrairement à Antony ou Sébastien, Alice se détourne du marché du travail artistique local, se justifiant du fait qu'aucun poste ne corresponde à sa vocation héritée des Beaux-arts : la production dans le champ de l'art contemporain. Un peu plus de six mois après son retour, la jeune femme propose ses services au rectorat. Elle est finalement recrutée comme professeure documentaliste dans un collège du département, en tant que vacataire dans un premier temps avant de devenir contractuelle.

Depuis 2010, elle occupe cette profession au gré de la vacance des postes, contrainte à des périodes d'inactivité qui peuvent atteindre plus de six mois. A l'occasion d'un entretien réalisé en 2014, Alice présente l'incertitude de cet emploi comme une caractéristique positive, qui lui permettrait de préserver sa "liberté », selon elle essentielle à nourrir son travail de création artistique. Un an plus tard, la jeune femme m'apprend que le rectorat ne lui a pas attribué de poste en cette rentrée scolaire, alors que le mois de septembre est déjà largement entamé. S'initie ainsi une longue période d'inactivité, durant laquelle Alice parvient de moins en moins à cacher l'appréhension qui l'envahit à l'idée de perdre définitivement cet emploi. En avril, soit près de 10 mois après la fin de son précédent contrat, la jeune femme est finalement contactée par le rectorat qui lui propose un remplacement dans un collège de la région, situé à près de 50 kilomètres. Malgré l'éloignement de l'établissement, le fait que le poste ne soit qu'à mi-temps (18h), et qu'en conséquence le salaire gagné équivaut au montant des indemnités chômage qu'elle touchait jusqu'ici, Alice accepte la proposition, justifiant cette décision par le fait qu'elle n'a d'autre choix que de « se faire bien voir par le rectorat ». Ce temps libéré ne lui 
permet toutefois pas de renouer avec une pratique artistique. A l'automne 2015, à l'occasion d'une conversation menée en aparté en marge d'une Assemblée Générale de l'association où nous sommes tous les deux bénévoles, la jeune femme m'apprend avoir abandonné ces projets en cours, affirmant «ne plus être dans le truc » depuis la rentrée.

Le cas d'Alice rend ainsi compte de la précarité et de l'incertitude qui gagnent les jeunes diplômées d'origine populaire (Palheta 2017) et/ou d'espaces en marge des grandes agglomérations (Orange 2017). Cette précarité résulte le plus souvent de salaires qui ne dépassent que rarement le SMIC, associés au fait que les temps partiels contraints sont monnaiecourante. Ces moyens économiques modestes s'accompagnent d'une grande incertitude concernant leur situation d'emploi, soit qu'elle soit liée au statut d'emploi (contrats à durée déterminée) soit que la structure qui les emploie voie son existence menacée, comme c'est le cas de nombreuses associations culturelles dans le contexte local. Le phénomène n'a rien de propre à Lergnes mais rend compte de certaines des métamorphoses de l'action publique, et tout particulièrement de l'accélération des politiques de réduction des dépenses publiques depuis la fin des années 2000 (Bresson 2016 ; Cottin-Marx et al. 2017 ; Gollac et Hugrée 2015), qui prend à Lergnes, comme au sein des autres villes moyennes en déclin, une importance exacerbée en raison de la grande dépendance de cet espace au tertiaire public (Béal et al. 2017). L'effritement de cette filière de promotion sociale concerne en particulier les derniers arrivés sur le marché de l'emploi (les personnes nées dans les années 1980, à l'instar des primoaccédants à l'université d'origine modeste dont il est ici question), plus fréquemment embauchés sous le statut de contractuel par les collectivités de la zone d'emploi que celles nées dans les années 1960 et $1970^{11}$.

\footnotetext{
${ }^{11}$ Les trois plus importantes collectivités lergnoises confondues, le taux de non-titulaires atteint $58 \%$ parmi les agents nés dans les années 1980, 21,2\% parmi ceux nés dans les années 1970 et 10,1\% parmi ceux nés dans les années 1960. Sources : bases des services ressources humaines du conseil départemental, de la commune de Lergnes et de l'agglomération de communes de Lergnes.
} 
La « lergnose »: habiter une ville moyenne en déclin

Le fait que ce retour se fasse dans une ville moyenne en déclin s'ajoute aux difficultés rencontrées sur le terrain de l'emploi. Cet aspect n'est pas anodin, il constitue au contraire un des éléments qui façonne la dimension subjective de la mobilité et produit le sentiment de déclassement. Pour le comprendre, rappelons à quel point l'image des villes petites et moyennes peut être négative et cantonnée aux marges inférieures de la hiérarchie des territoires. Cet état de fait, s'il s'est peut-être accentué à l'heure où la métropole mondialisée fait figure d'espace de référence (Wagner 2007 : 44-66), n'a rien de neuf dans le contexte français, façonné par plusieurs siècles de centralité économique et culturelle de Paris (Gravier 1947). Ce retour vient alors doublement contredire les aspirations sociales de ces étudiantes de première génération, à la fois car il vient rendre moins crédible l'intégration au marché du travail artistique mais aussi car il se fait à destination d'un espace dévalorisé. Je me propose maintenant d'en rendre compte en introduisant un nouveau cas, celui de Sandy, dont la représentation de Lergnes, lieu qui incarne pour elle la «lergnose », est largement partagée par les jeunes étudiants de retour, et plus particulièrement celles passées par des filières de production et de médiation artistiques.

Sandy présente la particularité d'avoir quitté Lergnes à 11 ans, ville d'où est originaire sa famille, pour s'installer en Guadeloupe, où son père, à l'origine animateur sportif dans un club d'athlétisme (il n'a pas le baccalauréat), est promu professeur d'EPS titulaire et où sa mère parvient à trouver un emploi de secrétaire ${ }^{12}$. En revanche, elle ne rompt jamais tout à fait avec sa famille et les quelques amis restés sur place et rentre ponctuellement pour y revoir cousins et cousines, oncles et tantes, ainsi que ses grands-parents. Bonne élève, elle s'inscrit en filière scientifique au lycée, tout en prenant une option facultative arts plastiques et obtient le

\footnotetext{
${ }^{12}$ Cette mobilité professionnelle ascendante du père par l'athlétisme fait de Sandy un cas particulier, dont les origines sociales sont plus favorisées que celles d'Alice ou d'Antony. Néanmoins, les origines populaires des deux parents, le fait qu'ils n'aient pas fait d'études supérieures, ou encore qu'ils soient tout à fait étrangers au champ de l'art contemporain, justifient le rapprochement avec les autres cas traités dans cet article.
} 
baccalauréat avec une mention « bien ». Elle passe les concours d'entrée aux écoles des Beauxarts et est reçue à celle de Marseille, où elle s'installe à la rentrée de l'année scolaire 20022003. A l'instar d'Alice, qui est au même moment aux Beaux-arts de Lyon, la scolarisation dans une école sélective et intégrative, où les pratiques culturelles et les sociabilités des étudiants sont strictement régulées par l'institution, participe à la réussite d'une familiarisation à la culture légitime et à conforter ses aspirations culturelles.

Cependant, elle aussi peine à y asseoir sa position. Tenue à l'obligation de faire un stage en troisième année, elle ne parvient pas à en trouver un à Marseille. Sur les conseils d'une de ses professeurs, qui sait que la jeune fille est originaire de Lergnes, elle sollicite un stage dans le centre d'art contemporain de sa ville d'origine, où elle fait la connaissance d'Antony, avec qui elle se mettra en couple un an plus tard. Sandy connaît des difficultés semblables à celles d'Alice une fois son diplôme de cinquième année obtenu : elle enchaîne plusieurs petits boulots précaires et incertains, notamment dans l'intermédiation culturelle, qui lui permettent de se maintenir dans cette ville, mais ne parvient pas à y exposer, ni même à produire de nouvelles pièces (elle n'a plus d'atelier). Elle postule en parallèle à de nombreuses résidences d'artiste, sans voir une seule de ses candidatures retenue. Un peu plus de deux ans après sa sortie de l'école, l'association qui l'embauchait dans le cadre d'un CAE (Contrat d'Accompagnement dans l'Emploi) pour créer et diffuser des porte-folios d'artistes ne peut renouveler son contrat. Cette éviction du marché du travail artistique marseillais la conduit à rentrer à Lergnes en 2011, où Antony la rejoint.

Ce retour est pensé dès le départ comme une manière de renouer avec une pratique artistique. Le couple trouve une petite maison en location, où Sandy dispose d'un atelier. Quelques mois plus tard, la jeune femme décroche un emploi de vendeuse à mi-temps dans une boutique d'objets design du centre-ville, qu'elle désigne comme «alimentaire », puis devient professeure d'arts appliqués contractuelle (également à temps partiel) à partir de la rentrée de 
2016. Si ce nouvel emploi correspond davantage aux aspirations culturelles de la jeune femme, cette dernière est, à l'instar d'Alice, critique à l'égard de la précarité (son salaire net avoisine alors les $800 €$ ) et de l'incertitude (la pérennité de cet emploi n'est pas garantie) qui caractérisent ce nouvel emploi. Ces difficultés d'accès au marché du travail artistique couplées aux conditions d'emploi précaires n'entachent pas dans un premier temps la vocation artistique de la jeune femme. Elle candidate à de nombreuses résidences d'artiste dans plusieurs grandes villes européennes, une nouvelle fois sans être retenue.

Lorsque je l'interroge à ce sujet dans le cadre d'un entretien en 2015, Sandy justifie cette envie de quitter Lergnes par le fait qu'elle ne parvienne pas à « décoller », c'est-à-dire à se positionner en tant qu'artiste-plasticienne dans le champ de l'art contemporain. Elle revient notamment sur les raisons qui la détourneraient de sa vocation et s'arrête longuement sur l'effet négatif du contexte local : la modestie des perspectives professionnelles qui s'offrent à elle, le manque de «diversité» de ses relations sociales au sein de l'espace local ainsi que la «lergnose » qui frapperait le territoire.

\footnotetext{
«Franchement, Lergnes j'aime bien. Si c'était pas aussi pénible de devoir batailler tout le temps pour sortir d'une espèce de beaufitude générale. Ça va pas devenir Lyon, on le sait bien, mais je trouve que la diversité... on n'a pas de diversité en fait : que des concerts de rock, toujours les mêmes gens. Il y a pas l'ébullition culturelle et sociale que tu retrouves dans d'autres villes et qui t'incite à produire [des œuvres d'art]. Je sais pas ce qu'il y a de moribond dans cette ville. C'est la lergnose !».
}

Sandy n'est pas la seule à dresser un tel constat, cette représentation négative est partagée par les étudiants de retour passés par les filières les plus distinctives et/ou les plus fortement inscrites dans la transmission d'une culture savante (lettres, arts, humanités), dont sont majoritairement issus les bénévoles associatifs rencontrés durant l'enquête. Cette stigmatisation de leur propre lieu de résidence procède de l'adhésion à un ordre symbolique légitime qui fait de Lergnes et des villes petites et moyennes «de province » des espaces repoussoirs, où sévirait une « beaufitude généralisée », à rebours de «l'ébullition culturelle et sociale » des grandes villes. Les effets de la «lergnose », c'est-à-dire de l'intériorisation de 
cette stigmatisation de leur espace de résidence, se conjuguent à ceux engendrés par l'incertitude et la précarité rencontrés au travail, pour susciter chez ces jeunes femmes un sentiment de déclassement. Celui-ci, dont Alice rend compte dans l'extrait qui suit, résulte ainsi du décalage entre les aspirations socio-résidentielles construites et consolidées tout au long de leur scolarité, celles de devenir artiste-plasticienne à Marseille et à Lyon dans les cas qui ont été présentés, et la réalité de leur position sociale et résidentielle actuelle :

\footnotetext{
«Un an de prépa, cinq ans de Beaux-arts, les stages et les boulots pourris, pour se faire envoyer chier au boulot. [...] Et puis être à Lergnes... Moi j'ai rien contre Lergnes, c'est juste l'ambiance générale. Des fois tu as l'impression d'être dans le vide, de parler à des murs, d'être au royaume de la médiocrité. »
}

\section{Contenir le sentiment de déclassement}

Les associations culturelles, une scène alternative de reconnaissance du capital culturel

Ce sentiment de déclassement peut cependant être compensé par l'investissement de scènes sociales remplissant la fonction d'« univers de consolation » (Poliak 2007). Il en est ainsi de certaines associations culturelles lergnoises, qui offrent à ces diplômées de retour une scène d'actualisation et de reconnaissance du capital culturel accumulé lors de l'émigration étudiante. Au moment où je les rencontre, Alice et Sandy sont ainsi toutes deux des militantes associatives s'investissant fortement dans leurs projets réciproques : Alice dans un local associatif où se mêlent concerts rock, expositions d'art contemporain et conférences ; Sandy dans une galerie associative d'art contemporain qu'elle a fondée en 2014 avec Antony, son compagnon d'alors rencontré en stage à Lergnes cinq ans plus tôt.

Né en 1984 et fils unique, Antony est originaire d'une petite commune ouvrière à proximité de Lergnes. Sa mère, qui a longtemps exercé le métier de couturière dans une usine de la région, est aujourd'hui au chômage ; elle compte désormais les quelques années qui la séparent de la retraite. Son père, quant à lui décédé, a été soudeur dans une usine située à 200 mètres du centre d'art contemporain où Antony travaille lorsque que je le rencontre en 2014. 
Le jeune homme s'inscrit en filière générale, poursuit en licence d'histoire de l'art à Dijon, et complète cette formation par un master professionnel « critique d'art». A l'issue de ses études supérieures, Antony cherche un emploi dans une grande ville française. Il multiplie alors les stages et les petits boulots afin d'y parvenir, en particulier au sein d'une prestigieuse galerie d'art contemporain du $6^{\text {ème }}$ arrondissement de Paris ${ }^{13}$, et décroche finalement un emploi de chargé de programmation dans un musée public dédié à l'art contemporain dans une grande agglomération française, à raison de $12 \mathrm{~h}$ par semaine. Après 18 mois à ce poste, tandis qu'aucune autre perspective professionnelle ne s'offre à lui, il est informé de la vacance d'un poste de chargé de programmation à temps plein dans le centre d'art où il a réalisé un stage en 2008 et fait la connaissance de Sandy. Il postule et voit sa candidature retenue, tandis que Sandy ne candidate pas, se justifiant a posteriori par le fait qu'elle préfère se concentrer sur ses activités de production artistiques.

C'est principalement cette opportunité, associée aux déboires professionnels rencontrés par Sandy à Marseille au même moment, qui motive le couple à élire domicile à Lergnes. Cette installation s'organise ainsi, dans le cas d'Antony tout au moins, avec un degré moindre de contrainte que ce qu'il est habituel d'observer dans les migrations retour des étudiantes de première génération. Elle est l'occasion pour le couple de créer une galerie d'art contemporain associative. Ce projet vise à investir un commerce de centre-ville fermé depuis de nombreuses années pour y installer un espace d'exposition en vitrine. Ce dispositif présente l'avantage de limiter les coûts : la galerie n'a pas à respecter les règles imposées aux Espaces Recevant du Public (ERP), de même qu'il n'est pas nécessaire de mobiliser une personne pour assurer une permanence, la visite de l'exposition se faisant depuis la rue. La boutique est prêtée

\footnotetext{
${ }^{13}$ L'accès à cette galerie lui est permis par la cooptation d'un artiste à la renommée internationale y exposant, également originaire de Lergnes, sur lequel Antony a réalisé son mémoire et qu'il a rencontré à cette occasion. Ce cas fait ainsi écho au constat d'une forte homophilie de genre dans les univers artistiques, au sein desquels la cooptation entre hommes est fréquente (Buscatto 2015).
} 
gracieusement par une femme à la retraite, qui ne cherche plus à louer son bien depuis des années, tandis que les frais d'aménagement de l'espace (2 $000 €)$ sont pris en charge par une levée de fond sur internet. La charge administrative (la communication, l'organisation, les relations avec les artistes, etc.) est assurée par Sandy, qui n'occupe pourtant officiellement aucune responsabilité au sein de l'association, afin de ne pas écarter la possibilité d'en devenir salariée une fois d'hypothétiques subventions obtenues ${ }^{14}$. Le poste de président revient à Antony, qui a également en charge la sollicitation d'artistes, disposant davantage que sa compagne de relations efficientes dans le champ de l'art contemporain.

Cette organisation n'est ainsi pas sans rappeler les «stratégies familiales de reproduction » analysées par Céline Bessière et Sybille Gollac (2020 : 56-60), dans la mesure où elle repose sur l'investissement de ressources inégalement distribuées au sein du couple (symboliques, relationnelles et économiques) dans un projet collectif de stabilisation de leurs positions au sein du champ de l'art contemporain. Elle permet dans un premier temps la réussite de l'initiative : en l'espace de 18 mois, 11 expositions sont organisées, dont certaines avec des artistes dont la renommée est internationale et d'autres suivant une trajectoire de consécration dans le champ. Les profits tirés de cet engagement sont partagés. Sandy y trouve d'abord l'occasion d'y exposer une nouvelle fois son travail tandis que la venue d'artistes permet aux deux de mettre en commun et d'actualiser leur capital social. Elle intègre ainsi peu à peu la petite bourgeoisie culturelle lergnoise, rencontre certains enseignants du lycée où elle travaille depuis 2016, qui soutiendront sa candidature auprès du rectorat. Par ailleurs, bénéficiant à la fois du temps et de l'espace pour produire de nouvelles œuvres ainsi que d'un lieu où les exposer, elle parvient à porter à nouveau le statut d'artiste-plasticienne au sein du groupe, sans

\footnotetext{
${ }^{14} \mathrm{Il}$ n'est en effet légalement pas permis de cumuler des responsabilités électives et un emploi rémunéré au sein d'une même association. En conséquence, les fonctions de trésorier et de secrétaire, bien que prises en charge par Sandy, sont officiellement assurées par un ami du couple, un professeur d'arts plastiques d'une quarantaine d'années.
} 
toutefois tirer de cette reconnaissance locale une opportunité d'entrée sur le marché du travail artistique.

L'accès à cet univers de consolation participe ainsi à rendre acceptable une « expérience subjective trop douloureuse du sous-emploi ou d'une précarité parée des atours de la bohème » (Dubois 2011 : 171). Il reste cependant fragile, du fait que ces associations culturelles, à plus forte raison lorsqu'elles sont d'implantation récente, ne survivent que grâce aux lourds investissements consentis par les membres bénévoles, en raison du refus des partenaires publics (l'Etat et les collectivités) d'apporter un soutien économique. Ainsi, moins de deux ans après sa création, l'association de Sandy et d'Antony met fin à ses activités, faute de subventions qui permettraient de dégager un salaire à Sandy, qui, «débordée » par ce travail bénévole et son emploi dans l'éducation nationale, n'a d'autre choix que d'abandonner le projet. L'association où s'engagent Alice et Sébastien suit exactement le même destin : faute de perspectives professionnelles pour les membres les plus impliqués dans le travail bénévole, les demandes de subvention essuyant des refus, ces derniers se retirent peu à peu de leurs activités associatives.

\section{Un repli sur le monde privé résidentiel}

Face à cette fermeture du marché du travail artistique, et à côté de cet univers de consolation que représentent les associations culturelles, plusieurs de ces jeunes diplômées d'école d'art, à l'instar d'Alice, investissent une autre scène sociale, le monde privé résidentiel. Au printemps 2016, près de 6 mois après la fin des activités de l'association où ils étaient tous deux bénévoles, le couple achète un ancien moulin en ruine dans une commune rurale à une trentaine de kilomètres de Lergnes. Cette acquisition est permise par les économies accumulées par Sébastien, qui a occupé un poste à responsabilité d'informaticien à Paris, avant de devenir membre et manageur d'un groupe de musique lergnois sous le statut d'intermittent du spectacle. Grâce au soutien des amis venus en nombre aider pour les travaux, les deux maisons mitoyennes qui composent le moulin sont habitables le printemps suivant. Cette installation désormais 
permanente contribue à éloigner encore davantage le couple de leurs engagements associatifs et de leurs pratiques artistiques, d'autant qu'elle s'accompagne de la grossesse d'Alice et du départ de Sébastien de son groupe de musique pour créer une auto-entreprise d'informatique.

A la fin de l'été 2017, je suis invité à une grande fête dans ce moulin, dont les derniers travaux sont presque achevés. La plupart des personnes présentes sont des anciens bénévoles de l'association culturelle. La fête est organisée dans la plus grande pièce de la bâtisse, où les combles ont été dégagés afin de faire apparaître les poutres. Au rez-de-chaussée se trouve une longue table avec un buffet où les premiers invités se servent déjà. Je discute longuement avec Sébastien, qui me fait visiter la maison. Ce tour des lieux se prolonge par une visite de l'étage, des pièces du rez-de-chaussée (cuisine, salles de bain, chambres), et de la maison adjacente qui n'a pas encore été rénovée. Nous nous arrêtons avec Sébastien devant chaque objet et élément auquel il juge bon d'associer une courte présentation. Ainsi, j'apprends que telle pièce est recouverte de «tommettes du coin », que le crépis de telle autre a été retiré par lui-même et quelques amis afin de laisser les « pierres apparentes », ou encore que des fenêtres triple vitrage ont été posées ici et là. S'incarnent ici, dans les objets ou dans les matériaux de construction, certaines des dispositions à la distinction : les préoccupations écologiques et esthétiques que révèle le choix d'un poêle à granulés, ou celui de l' «authenticité » des poutres et des pierres apparentes, comme si cet espace privé résidentiel se faisait le support de leurs aspirations culturelles déçues.

Ce désengagement de l'espace public pour l'espace privé s'inscrit en premier lieu dans le processus de transition des différentes étapes du cycle de vie. Alice et Sébastien, alors respectivement âgés de 32 et 39 ans, ont ainsi leur premier enfant à un âge relativement tardif, de manière semblable à ce qui s'observe parmi les jeunes diplômés de retour à Lergnes, et plus généralement en France chez les titulaires d'un diplôme du supérieur (Volant 2017). De même, ils appartiennent à une tranche d'âge où les chances d'être accédants à la propriété augmentent 
significativement en France (Bugeja 2011 : 54), à plus forte raison encore à Lergnes où la valeur foncière des logements est relativement faible et où le retour qualifié des jeunes diplômés va souvent de pair avec des projets d'achat immobilier. Pour autant, cette soudaine stabilisation familiale et résidentielle répond également à une fermeture du champ des possibles sur le marché du travail artistique. Mais une telle voie de sortie n'est pas à la portée de tous ; elle nécessite au préalable une stabilité conjugale ainsi qu'un patrimoine économique (dont Sébastien disposait, ayant épargné sur ses « gros salaires » des « années parisiennes »), ou tout au moins les garanties nécessaires à l'obtention d'un prêt auprès d'une banque. Elle n'est par exemple pas envisageable pour Sandy et Antony, qui ne disposent ni des ressources économiques (ils n'ont aucun apport) ni d'une stabilité professionnelle et conjugale suffisante pour y parvenir. Ainsi, dès notre premier entretien en 2015, la jeune femme me fait part des incertitudes qui pèsent sur les projets professionnels et résidentiels du couple, qu'elle associe à leur manque d'argent mais aussi à l'investissement d'Antony dans «sa carrière », qui le conduira, dit-elle, à postuler à «moyen terme» sur "quelque chose avec plus de responsabilités, loin d'ici ». Non sans une certaine amertume, elle anticipe le dilemme auquel elle pourrait être confrontée, rester à Lergnes, au détriment de leur relation, ou «suivre » Antony, au risque d'accentuer encore davantage l'asymétrie de leurs positions respectives sur la scène professionnelle : «même si je suis mobile, je me vois mal suivre et faire des petits jobs, c'est pas comme ça que j'envisage ma vie ». Faute d'être en mesure de compenser pleinement leurs désillusions sur la scène associative ou sur celle du monde privé résidentiel, le couple traverse alors une grave crise, qui va conduire à leur séparation et au départ d'Antony pour Paris, comme l'avait partiellement anticipé Sandy un an plus tôt.

\section{Une séparation : celui qui part et celle qui reste}

Afin de comprendre les ressorts de cette séparation, il est nécessaire de rappeler que leur galerie associative s'inscrit à l'origine dans une stratégie conjugale d'ascension qui vise à 
«faire décoller» Sandy, en offrant à la jeune femme un lieu d'exposition et de rencontre avec des artistes et professionnels de l'art contemporain invités par Antony. Cette inégalité d'investissement en capital social instaure de fait une inégalité dans leur relation qui, à mesure que s'éloigne la perspective d'infléchir la bifurcation de leurs trajectoires, va être de plus en plus mal vécu par Sandy. En effet, si la jeune femme parvient à exposer son travail dans ce local associatif, sa légitimité d'artiste-plasticienne peine à être reconnue en dehors de l'espace local, si bien que cette expérience ne lui ouvre pas les portes des galeries et résidences d'artistes des grandes villes. A l'occasion des vernissages auxquels je participe, les artistes et critiques d'art allochtones présents, dans leur grande majorité des amis et anciens collègues de son compagnon, forment des groupes de discussion auxquels les membres de la petite bourgeoisie locale participent peu, et au sein desquels Sandy se trouve le plus souvent en retrait. Ce fait n'a pas échappé à la jeune femme qui, par exemple, dans un café à l'issue d'un de ces vernissages, m'explique s'être retenue de ne pas « péter les plombs » face à un artiste parisien jugé « hautain et méprisant », qui ne l'aurait sollicitée qu'au sujet des aspects techniques de son installation, la plaçant ainsi dans un rôle qu'elle qualifie d' «assistante ». Près d'un an après leur rupture, en novembre 2017, elle m'explique à l'occasion d'une discussion informelle avoir initié cette séparation en raison de la souffrance engendrée par le fait d'être vue comme «la copine d'Antony », assignation genrée qui, comme pour Alice lorsqu'elle sortait des Beaux-arts, vient fragiliser son statut d'artiste en le rendant tributaire des ressources de son conjoint (Buscatto 2008 : 97). Elle m'expliquera notamment avoir ressenti un «étouffement », du fait de la stagnation de ses perspectives professionnelles dans le champ de l'art contemporain tandis que son compagnon y confortait sa position.

Leur séparation, survenue à l'hiver 2016, va alors brusquement accélérer la bifurcation redoutée de leurs trajectoires, en réaffectant individuellement les ressources investies dans cette 
galerie d'art, au détriment de Sandy ${ }^{15}$. Moins d'un mois après cette séparation, Antony démissionne du centre d'art contemporain où il travaillait et trouve immédiatement un nouvel emploi, comme responsable d'une galerie d'art contemporain à Paris. Il se sépare ainsi des fondements de sa vie lergnoise : Sandy, la maison qu'il partageait avec elle (en location) et son poste de chargé de programmation dans ce centre d'art contemporain en périphérie de Lergnes. Un peu moins d'un an plus tard, je rencontre le jeune homme à Paris. Il m'apprend qu'il entretient une nouvelle relation amoureuse avec une jeune artiste américaine et qu'il s'est installé dans le Nord-est de la capitale. A regarder de près la trajectoire et les ressources d'Antony, cette nouvelle étape parisienne n'est pas surprenante. Le jeune homme compte en effet parmi les mieux dotés des diplômés de retour que comptent ces deux associations culturelles, tout particulièrement en ressources relationnelles extra-locales, du fait de son passage dans un musée d'art contemporain lyonnais et de stages distinctifs réalisés à Paris.

Depuis cette rupture, Sandy ne trouve plus le temps de «produire», se justifiant par le fait que son service de contractuel atteigne depuis la rentrée de 2017 l'équivalent d'un tempsplein. Par ailleurs, le réseau d'Antony dont elle pouvait bénéficier se ferme alors, de la même manière que celui des femmes musiciennes de jazz interrogées par Marie Buscatto (2015 : 143) qui se séparent de leur conjoints. Suite à cet épisode, elle ne me fera d'ailleurs plus état de ses espoirs d'intégrer le marché du travail artistique. C'est dans ce contexte que s'opère chez elle un réajustement des aspirations. Elle m'apprend à l'automne, un an après sa rupture avec Antony, qu'elle prépare le concours du CAPET (Certificat d'Aptitude au Professorat de l'Enseignement Technique) d'arts appliqués. Toujours célibataire et sans autre perspective professionnelle, ce désir de concours va, en février 2018, se muer en injonction pressante, alors

\footnotetext{
${ }^{15} \mathrm{Si}$ les ressources dont il est question ici sont d'abord relationnelles et symboliques, il convient de faire un parallèle avec les analyses de Céline Bessière et de Sybille Gollac, qui montrent que les séparations dans les couples hétérosexuels se traduisent généralement par un partage inégal du patrimoine économique, au détriment des femmes $(2020: 83-85)$.
} 
qu'elle apprend que son poste de professeure d'arts appliqués est supprimé pour la rentrée de septembre, de même que l'ensemble des emplois contractuels de ce lycée.

A partir du cas de jeunes diplômées d'écoles des Beaux-arts, cet article a proposé une analyse longitudinale de la production et de la gestion du désajustement entre les mobilités objectives et les aspirations subjectives. Si, dans leur cas, ce phénomène apparaît exacerbé et s'inscrit dans la durée, c'est d'abord en raison des conditions particulières de scolarisation qu'elles ont connues, au sein d'écoles d'art sélectives disposant d'un fort pouvoir d'acculturation. La socialisation qu'opère l'institution a d'abord trait à certains registres de la culture légitime, en particulier de ceux en cours dans le champ de l'art contemporain (culture savante, artistique et littéraire), mais plus généralement à un style de vie au sens où l'entend Pierre Bourdieu, c'est-à-dire à un ensemble de pratiques, de normes et de croyances, qui les portent à adopter les critères de perceptions de leur milieu d'élection. Dès la sortie de l'école, leurs aspirations à devenir artistes-plasticiennes vont pourtant se heurter aux droits d'entrée du marché du travail artistique, qui, comme le montrent de nombreuses recherches ${ }^{16}$, et comme cet article l'a rappelé, portent préjudices aux jeunes de classes populaires et aux femmes.

Cet échec s'accompagne d'un retour à l'espace d'origine, une ville moyenne en déclin. Si plusieurs recherches défendent l'idée que certains espaces de résidence sont plus propices que d'autres à des mobilités sociales et professionnelles ascendantes (Dherbécourt 2015), les analyses présentées ici montrent que les déplacements géographiques participent également à la construction de la mobilité subjective. Ces diplômées, qui aspirent à intégrer un marché du travail artistique dont les institutions de régulation et les agents dominants sont concentrés dans

\footnotetext{
${ }^{16}$ Voir notamment les travaux de Marie Buscatto, Vincent Dubois, Gérard Mauger, Delphine Naudier, Dominique Pasquier, Hyacinthe Ravet et Pierre-Emmanuel Sorignet déjà cités.
} 
les grandes agglomérations et en particulier à Paris, se montrent en effet particulièrement promptes à reconnaître l'assignation des villes moyennes au bas de la hiérarchie symbolique des territoires. S'il convient de ne pas essentialiser les appartenances territoriales, dont on a pu montrer que les effets s'articulaient à ceux de l'origine sociale, du genre, ou encore de la situation matrimoniale, rentrer à Lergnes revient, pour ces jeunes femmes, à renoncer partiellement à leurs aspirations et à connaître la «lergnose ».

Ce sentiment peut cependant être contenu par l'investissement de différentes scènes de compensation, dont on a pu mesurer l'importance mais aussi l'instabilité. Le réinvestissement des aspirations déçues sur la scène associative se heurte ainsi à la fragilisation de cette dernière, en raison de l'absence de soutien des partenaires publics. Le repli sur un monde privé conjugal, dans lequel s'engagent avec succès Alice et Sébastien ${ }^{17}$, est quant à lui tributaire de conditions dont ne disposent pas Sandy et Antony : des ressources économiques tout d'abord, mais aussi un horizon professionnel, familial et résidentiel commun. Or, si dans le cas des deux couples les hommes sont mieux dotés que les femmes en capital économique (Sébastien), culturel et social (Antony), ces différences prennent une importance telle dans le cas de Sandy et d'Antony qu'elles entravent un réajustement des aspirations à l'échelle conjugale. La séparation qui en découle vient alors entériner une bifurcation des trajectoires initiée des années plus tôt ${ }^{18}$.

Enfin, les résultats présentés dans cet article invitent à ne pas séparer l'analyse des mobilités professionnelles de celle des conditions sociales, géographiques, institutionnelles et politiques qui les déterminent, comme le préconisent Julie Pagis et Paul Pasquali (2016 : 114).

\footnotetext{
17 A l'automne 2020, ils habitent toujours ce moulin réaménagé et ont un deuxième enfant. Les deux semblent s'être durablement détournés de leurs aspirations artistiques et des sociabilités de la petite bourgeoisie culturelle lergnoise. Alice continue d'exercer l'emploi de professeure documentaliste en tant que contractuelle tandis que Sébastien a créé une petite entreprise d'informatique dont il est le seul salarié.

18 Antony est désormais commissaire d'exposition au sein d'un prestigieux musée d'art contemporain parisien. Sandy a quant à elle réussi le concours du CAPET d'arts appliqués et a été mutée dans une ville moyenne de l'est de la France, mais a demandé sa mutation à Lergnes où elle entretient une relation avec un maraîcher depuis près d'un an.
} 
Que l'on considère les obstacles rencontrés à intégrer le marché du travail artistique, la précarité et l'incertitude sur le terrain de l'emploi public, ou bien encore la fragilisation des scènes de compensation, il apparaît que ces phénomènes ne sont pas étrangers aux effets de l'intensification des politiques de réduction des dépenses publiques initiée par la Révision Générale des Politiques Publiques de 2007, particulièrement forts au sein des espaces en marge des grandes agglomérations (Chouraqui 2020 : 27). L'analyse des mobilités professionnelles ne peut ainsi faire l'économie d'un examen attentif des conditions dans lesquelles le diplôme, et plus généralement les ressources délivrées par l'institution scolaire, les rendent possible ou au contraire les entravent, à plus forte raison au sein des univers professionnels dont l'accès repose sur des ressources relationnelles et des formes incorporées et non-certifiées de capital culturel.

\section{Bibliographie}

Béal, Vincent, Anaïs Collet, James DeFilippis, Richard E. Ocejo et Max Rousseau. 2017. "Villes en décroissance », Métropolitiques, http://www.metropolitiques.eu/Villes-endecroissance.html: consulté le 28 mars 2017.

Beaud, Stéphane. 2002. " $80 \%$ au bac »... et après? Les enfants de la démocratisation scolaire. Paris, la Découverte.

Beaud, Stéphane. 1997. «Un temps élastique », Terrain, nº 29 : 43-58.

Bessière, Céline et Sibylle Gollac. 2020. Le genre du capital. Paris, la Découverte.

Bresson, Maryse. 2016. «Le travailleur non titulaire dans la fonction publique: une figure emblématique des marges de l'emploi », Revue Française de Socio-Économie, no 17 : 65-83.

Bugeja, Fanny. 2011. "Les inégalités d'accès à la propriété et leurs déterminants institutionnels », Revue française de sociologie, vol. 52, $\mathrm{n}^{\circ} 1:$ 37-69.

Buscatto, Marie. 2015. "La féminisation du travail artistique à l'aune des réseaux sociaux », Sociologie de l'Art, vol. 23-24, nº 1 : 129-152.

Buscatto, Marie. 2008. «Tenter, rentrer, rester: les trois défis des femmes instrumentistes de jazz », Travail, genre et sociétés, $\mathrm{n}^{\circ} 19, \mathrm{n}^{\circ} 1: 87-108$.

Chauvel, Louis. 1998. "La seconde explosion scolaire: diffusion des diplômes, structure sociale et valeur des titres "), Revue de l'OFCE, vol. 66, n $1:$ 5-36.

Chouraqui, Julie. 2020. «Les réformes et l'État et la transformation des services publics : des tendances internationales au local, quels effets pour les territoires? », Annales de géographie, $\mathrm{n}^{\circ} 732, \mathrm{n}^{\circ} 2: 5-30$. 
Convert, Bernard. 2010. «Espace de l'enseignement supérieur et stratégies étudiantes », Actes de la recherche en sciences sociales, $\mathrm{n}^{\circ} 183, \mathrm{n}^{\circ} 3: 14-31$.

Cottin-Marx, Simon, Matthieu Hély, Gilles Jeannot et Maud Simonet. 2017. «La recomposition des relations entre l'État et les associations: désengagements et réengagements », Revue française d'administration publique, $\mathrm{n}^{\circ} 163$ : 463-476.

Dherbécourt, Clément. 2015. «La géographie de l'ascension sociale », La note d'analyse de France Stratégie, no $36: 1-8$.

Dubois, Vincent. 2011. La culture comme vocation. Paris, Raisons d'agir.

Eckert, H. 2011. « Les diplômes et leur valeur », in La société des diplômes, La dispute, Paris, France : 51-66.

Erb, Louis-Alexandre. 2018. « Disciplines du diplôme de master et insertion professionnelle selon le genre », Éducation et formations, ${ }^{0} 98: 85-111$.

Gollac, Sibylle et Cédric Hugrée. 2015. «Avoir trente ans dans le secteur public en 1982 et en 2002 les transformations d'une filière de promotion sociale par le diplôme ", Revue française d'administration publique, $\mathrm{n}^{\circ} 153: 23-43$.

Gravier, Jean-François. 1947. Paris et le désert français : décentralisation, équipement, population. Paris, Le Portulan.

Guéraut, Élie et Fanny Jedlicki. 2021. «L'émigration étudiante des filles du « coin» : entre émancipation sociale et réassignation spatiale », Travail, Genre et sociétés, nº 46 (à paraître).

Hugrée, Cédric. 2011. «Aux frontières du «petit» salariat public et de son encadrement : de nouveaux usages des concours? », Travail et Emploi, $\mathrm{n}^{\circ} 127: 67-82$.

Hugrée, Cédric. 2010. « «Le CAPES ou rien?» », Actes de la recherche en sciences sociales, $\mathrm{n}^{\circ} 183, \mathrm{n}^{\circ} 3: 72-85$.

Laferté, Gilles et Florence Weber. 2019. «Introduction», in Territoires, culture et classes sociales, Paris, France, Éditions Rue d’Ulm : 7-26.

Mauger, Gérard. 2007. « Droits d'entrée. Quelques enseignements d'une enquête collective », in Droits d'entrée. Modalités et conditions d'accès aux univers artistiques., Paris, France, Les Editions de la MSH : 251-268.

Mauger, Gérard. 2006. «Le capital spécifique », in L'accès à la vie d'artiste, Paris, France, Editions du Croquant: 237-253.

Mayaud, Isabelle et Laurent Jeanpierre. 2020. «Destinies of artistic activity: visual artists' plural forms of employment and trade-offs in a French region », Sociologica del Lavoro : 125144.

Merle, Pierre. 2009. La démocratisation de l'enseignement. Paris, la Découverte. 
Naudier, Delphine. 2014. «Les modes d'accès des femmes écrivains au champ littéraire contemporain ", in Droits d'entrée. Modalités et conditions d'accès aux univers artistiques, Editions de la Maison des sciences de l'homme : 191-214.

Orange, Sophie. 2017. " "Celles qui restent”. La fausse inertie des jeunes diplômées du coin », in Une génération sacrifiée? Jeunes des classes populaires dans la France désindustrialisée, Paris, France, Editions de la rue d'Ulm : 113-126.

Pagis, Julie et Paul Pasquali. 2016. «Observer les mobilités sociales en train de se faire », Politix, $\mathrm{n}^{\mathrm{0}} 114$ : 7-20.

Palheta, Ugo. 2017. «Espoirs scolaires et déboires professionnels des filles d'origine populaire », in Une génération sacrifiée? Jeunes des classes populaires dans la France désindustrialisée. Paris, Editions de la rue d'Ulm : 99-112.

Pasquali, Paul. 2010. "Les déplacés de 1'«ouverture sociale»», Actes de la recherche en sciences sociales, $\mathrm{n}^{\circ} 183, \mathrm{n}^{\circ} 3: 86-105$.

Pasquier, Dominique. 1983. «Carrières de femmes : l'art et la manière », Sociologie du travail, vol. $25, \mathrm{n}^{\circ} 4: 418-431$.

Poliak, Claude F. 2007. "Pratiques et univers de consolation. Les écrivains amateurs », in Droits d'entrée. Modalités et conditions d'accès aux univers artistiques., Paris, Les Editions de la MSH : 215-250.

Ravet, Hyacinthe. 2006. «L'accès des femmes aux professions artistiques. Un double droit d'entrées dans le champ musical ", in L'accès à la vie d'artiste. Sélection et consécration artistiques : 151-176.

Siblot, Yasmine, Marie Cartier, Isabelle Coutant, Olivier Masclet et Nicolas Renahy. 2015. Sociologie des classes populaires contemporaines, Paris, Armand Colin.

Sorignet, Pierre-Emmanuel. 2016. « «Si j'arrête de danser, je perds tout»», Politix, $\mathrm{n}^{\mathrm{o}} 114: 121-148$.

Truong, Fabien. 2015. Jeunesses françaises : Bac +5 made in banlieue, Paris, France, la Découverte.

Volant, Sabrina. 2017. «Un premier enfant à 28, 5 ans en $2015: 4,5$ ans plus tard qu'en 1974 », Insee Première, $\mathrm{n}^{\mathrm{0}} 1642$.

Wagner, Anne-Catherine. 2007. Les classes sociales dans la mondialisation. Paris, la Découverte.

Weber, Florence. 1995. «L'ethnographie armée par les statistiques », Enquête, nº 1 : 153-165. 